\title{
MAPMT H7546B Anode Current Response Study for ILC SiD Muon System Prototype
}

\author{
A.Dyshkant, Member IEEE, G.Blazey, K.Francis, D.Hedin, V.Zutshi \\ Northern Illinois University, DeKalb, IL 60115, USA \\ H.Fisk, C.Milstene \\ Fermilab, Batavia, IL 60510, USA \\ R.Abrams \\ Indiana University, Bloomington, IN 47405, USA \\ (Submitted to IEEE Transaction on Nuclear Science)
}

\begin{abstract}
The proposed Silicon Detector (SiD) concept for the ILC has barrel and end cap muon systems. An SiD scintillator based muon system prototype has 256 strips and was constructed from extruded strips, WLS fibers, clear fibers, and multianode photo multiplier tubes (MAPMT) Hamamatsu H7546B. Six MAPMTs were used. As a first step to understand strip output, the response of every anode to a given brightness of light and applied voltage must be measured. For the test, a custom made light source was used. Each MAPMT output was measured independently. The anode currents were measured at constant (green) input light brightness and the same photocathode to anode voltage $(800 \mathrm{~V})$. The anode currents have a wide spread; for all tubes the maximum value is 5.23 times larger than the minimum value. The MAPMT cross talk was measured for one of the central inputs. The maximum cross talk value is about $4.9 \%$. The average cross talk for the nearest four neighboring channels is $3.9 \%$, for the farthest four is $1 \%$. To assure the reproducibility and repeatability of the measurements, the double reference method was used.
\end{abstract}

\section{Introduction}

The proposed SiD [1] for the International Linear Collider (ILC) has barrel and end cap muon systems (Fig.1). A $5.7 \mathrm{~m}$ long barrel will start outside of the calorimeters and the 5T superconductive magnet at a radius of $3.5 \mathrm{~m}$. The solenoid flux return is divided into 23 layers of $10 \mathrm{~cm}$ steel in an octagonal geometry. The muon detectors will be inserted between the plates.

A SiD scintillator based prototype installed in the Fermilab Meson Test Beam Facility (MTBF) has 256 strips. The prototype was constructed of extruded scintillating strips, WLS fibers, clear fibers, and multianode photo multiplier tubes (Fig.2) [2]. The scintillating strips were extruded with grooves for WLS fibers and titanium dioxide white coating. Kuraray Y-11 multicladed $1.2 \mathrm{~mm}$ outer diameter WLS fibers were thermally spliced to $1.2 \mathrm{~mm}$ outer diameter clear fibers WLS fiber ends were polished and one end was aluminum sputtering mirrored. Then WLS fiber was glued in the strip groove. The strips were formed in a layer with fibers assembled into a "cookie". Up to 64 fibers in one cookie were connected to a Hamamatsu tube H7546B [3].The layers were assembled in a module. Six MAPMTs were used. The MAPMT tubes have the following labels: S+, S-, D+(a), D+(b), D-(a), and D-(b).The module was first tested in 2006.

To clarify a comparison of strip responses to a beam particle, it was essential to apply the relative anode current correction to a particular strip's output. 


\section{Material and methods}

PMT anode response is sensitive to input light brightness, quantum efficiency of the photocathode, and gain, which depends on the applied voltage. Even in the case when a tube operates in complete darkness, a small amount of current flows through the photomultiplier (dark current). Usually gain is defined as the ratio of the anode current to the photoelectric current from the photocathode. In the case of stable gain, the output current response of a particular anode is usually different from a neighboring channel because of differences in the local quantum efficiency of the photocathode.

As a first step to understanding each strip's output, the response of every anode to a given input light brightness and applied voltage must be measured. The brightness of the light should be high, but not so high as to saturate MAPMT channels. For an accurate measurement, the PMT anode current response to the given input light brightness should be much higher than the dark current. For the comparison of different photomultiplier anode currents the irradiating light and the supplied voltage should be of reasonable values and stable during all anode current measurements. We used the company recommended value for anode to cathode supplied voltage, which is $800 \mathrm{~V}$.

For the following test, a custom made light source was used. To make the test light emission spectrum similar to the strip light, the custom made source also has scintillator and WLS fiber. The source consists of a $10 \mathrm{~mm}$ thick $10 \times 10 \mathrm{~cm}^{2}$ plate cast scintillator from EJ-200 [4] (similar to BC-408 or Pilot F) with two key shaped milled grooves, two Kuraray Y-11 [5], multicladed, $1.2 \mathrm{~mm}$ outer diameter, and $1.01 \mathrm{~m}$ long WLS fibers, two layers of Tyvek [6] wrapping, and a radioactive source on the top (5 mCi Sr-90 with housing) (Fig.3). The length of the fiber out of the scintillator is about 0.9 $\mathrm{m}$, which helps to reduce or avoid cladding light at the fiber end. During tests, insertion of the WLS fiber end into different inputs occurs more than four hundred times. The fiber end can be damaged and the cladding in particular. The modified or damaged cladding influence will be minimized if the light is in the core which will occur for longer fiber.

The light emission spectrum at the WLS fiber end is defined by the emission spectra of the Y11 material itself and the fiber length. Because the fiber attenuation is wavelength dependent, the one meter long Y11 WLS fiber has the maximum of relative intensity at about $500 \mathrm{~nm}$.

For the relative comparison of channel response the measurement conditions should be the same during all tests. To be sure of the same conditions, a double reference method was used [7]. The mirrored end of both WLS fibers were permanently connected (inserted, not glued) to the source-scintillator. The other ends of the WLS fibers were connected to the MAPMT assembly (Fig.4). A template (Fig.5a) was used to connect WLS fibers to the photocathode (Fig.5b). The template's hole position corresponds to the center of a particular photocathode channel which has a $2 \times 2 \mathrm{~mm}^{2}$ active area and was defined using the company catalog drawing [3]. One of the fibers was connected to channel 57 (Table 1) in the same position for each particular tube and was used for reference measurements. The second fiber was used to deliver the input light to each channel under test and for control measurements in channel 64 (see Table 1) also. The 
control measurements were performed after each row of eight channels was tested. The control measurements verified that the amount of input light was constant. The reference measurements were performed before the particular channel test and verified that the measurement system, including the MAPMT under test, was in the same condition. If the reference measurement values were repeatable with accuracy better than one percent for each particular MAPMT, the measurements performed between them were accepted.

Each MAPMT output was measured independently (Fig.6). So, for each input channel cross talk in sixty three outputs in the tube can also be measured. The cross talk is the output current in any channel which was not the channel where the WLS fiber with permanent input light is connected. The measurements were performed in a light tight box at a room temperature of about $+22^{\circ} \mathrm{C}$ on average. The setup in Fig. 7 was used.

To make the result transparent, the usual Hamamatsu mapping from the catalog was used (Table 1). Because channel 57 was used for the reference measurements, the neighboring channels 49,50 , and 58 do not have references because of cross talk. When channel 57 was being tested, it also did not have a reference.

\section{Results}

The first measurement goal was to verify that the brightness of the custom made light source did not saturate the anode current. The WLS fiber was permanently connected to the same MAPMT channel. The light brightness was reduced with sheets of plastic between the radioactive source and scintillator (Fig.8). Because there was no indication of output saturation, there was no additional adjustment of the light flux.

The anode currents measured for the MAPMT S+ are shown in Fig.9. The S+ tube provides an example of a wide variety of anode currents in each row. The minimum current was observed in channel \#01, the maximum in \#54. For these two channels the anode currents were measured in a range from 600 to $960 \mathrm{~V}$. The anode current dependences from applied voltage are in Fig.10. Because the shape of current-voltage characteristic was the same, the other measurements were performed at $800 \mathrm{~V}$ only.

The anode current average value was different for each tube. As an example, the normalized (to the average value) responses of MAPMT D-(b) are in Fig.11.

Because of a few percent deviation in the reference and the control measurements, the anode output current response of any channels can be directly compared. The average anode current for all six tubes was calculated. All tube anode currents normalized to this average value are in Fig.12. Parameters for each MAPMT anode current distribution are in Table 2.

MAPMT D-(b) cross talk was measured for one of the central inputs (channel \#37, Table 1). In this case all 64 anode currents were measured. The ratios of each anode current value to the anode current in channel \#37 are in Table 3. The MAPMT D-(b) cross talk values in column \#5 are in Fig.13.

Finally, the anode responses of MAPMT D+(a) and D+(b) measured at green light (about $500 \mathrm{~nm}$ ) were compared with Hamamatsu [3] factory measurements at blue light (about $400 \mathrm{~nm}$ ). The Hamamatsu final test sheet accompanying the tubes indicates anode uniformity in units normalized to the maximum value for each tube. For each channel, the anode current produced by a light flux of a tungsten lamp at $2856 \mathrm{~K}$ passing through a blue filter (Corning CS 5-58) was measured. The light flux was adjusted to an appropriate 
level and the applied voltage was $800 \mathrm{~V}$. The ratios of green light response output to the blue light response for D+(a) are in Fig.14. For D+(b) the ratios are in Fig.15.

\section{Conclusion}

The MAPMT anode currents were measured at constant green (about $500 \mathrm{~nm}$ ) input light brightness and the same photocathode to anode voltage $800 \mathrm{~V}$. The anode currents have a wide spread. For all tubes the maximum value is 5.23 times larger than the minimum value.

For individual MAPMT the results are in a good agreement with Hamamatsu anode uniformity factory measurements.

The MAPMT D-(b) cross talk was measured for one of the central inputs. The maximum cross talk value is about $4.9 \%$. The average cross talk for the nearest four neighboring channels is $3.9 \%$ and for the farthest four is $1 \%$.

The application of relative anode responses to a particular strip's output removes the effect of the PMT itself and clarifies a comparison of strip responses to a beam particle.

\section{Acknowledgement}

The authors would like to thank Pill Stone for preparing the template.

\section{References}

[1] M.Breidenbach, “SiD- a concept for ILC”, LCFOA-2006.

[2] R.J.Abrams “Test of a Pre-Prototype ILC Muon Detector Module”, SLAC R772 eConf: C050318, 2005, p.742.

[3] Hamamatsu Photonics K.K., Electron Tube Center, 314-5, Shimokanzo, Toyooka-village, Iwata-gun, Shizuoka-ken, 438-0193, Japan.

[4] Eljen Technology 2010 E. Broadway, Sweetwater, Texas 79556, United States

[5] Kuraray America Inc., 200 Park Avenue, NY 10166, USA.

[6] Du Pont Co. E.I.Du Pont de Nemours\&Co., Fibers Department, Chestnut Run Plaza, PO Box 705, Wilmington, DE 19880-0705; http://www2.dupont.com/Tyvek_Construction/en_US/products/commercialwrap.html

[7] A.Dyshkant, D.Beznosko, G.Blazey, E.Fisk, E.Hahn, V.Rykalin et al., "Quality Control Studies of Wavelength Shifting Fibers for a Scintillator-Based Tail Catcher Muon Tracker for Linear Collider Prototype Detector,” IEEE, vol. 53, 2006, p. 3944.

[8] DuPont Corp., Product Description, [Online]. Available: http://heritage.dupont.com/floater/fl_delrin/floater.shtml

\section{Questions}

\section{dyshkant@nicadd.niu.edu}




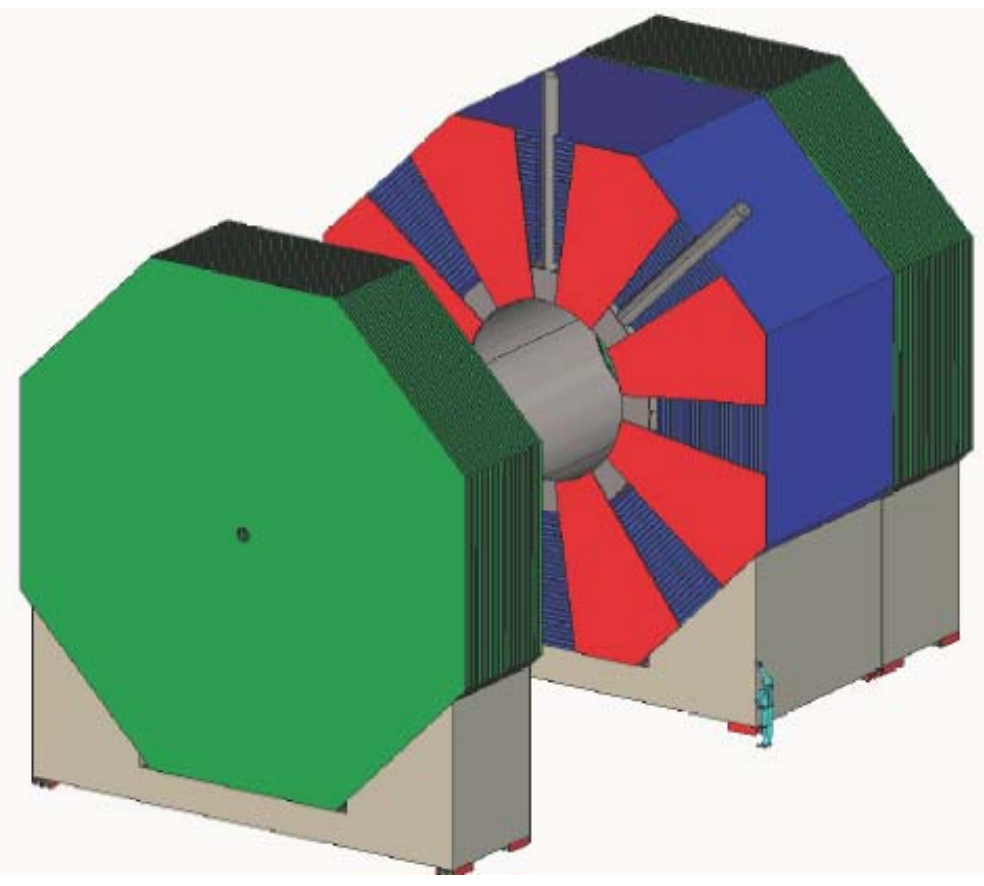

Fig.1. The proposed SiD for the ILC has barrel and end cap muon systems.

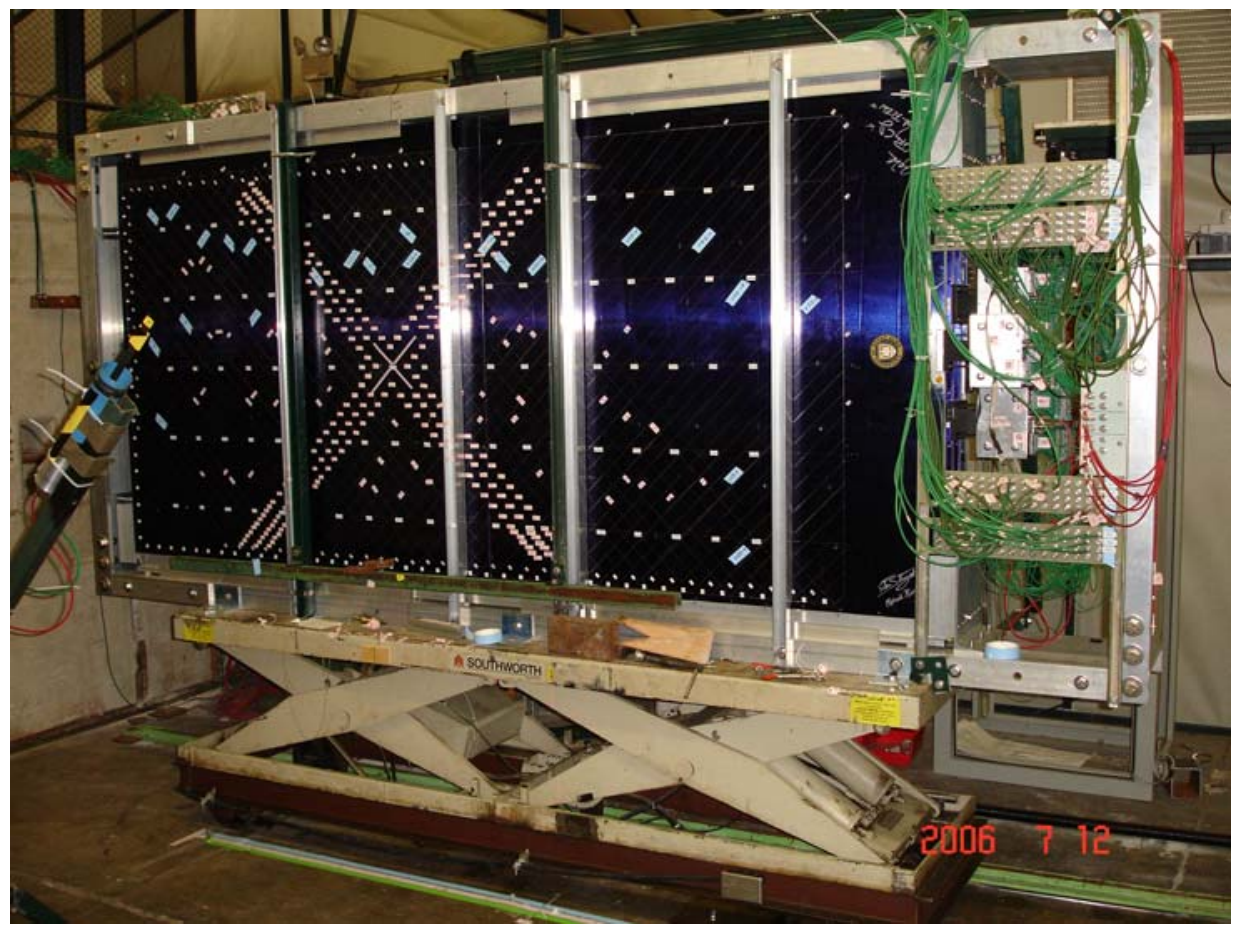

Fig.2. A SiD scintillator based muon system prototype installed at Fermilab in 2006. 


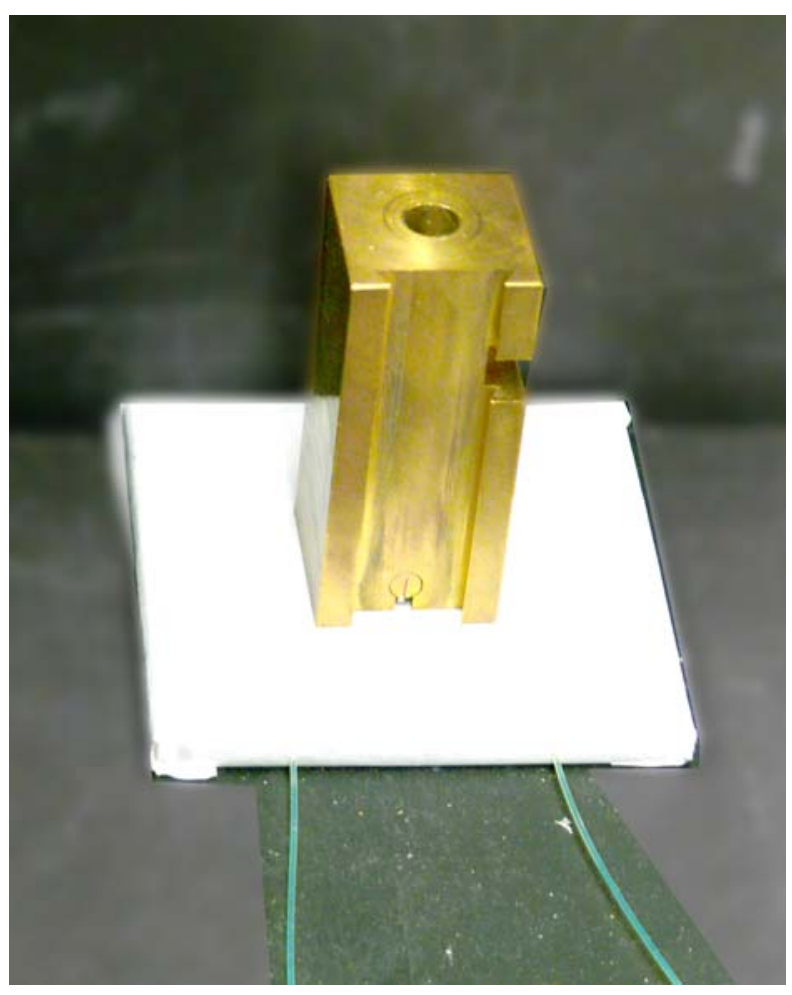

Fig.3. A custom made light source used in the test.

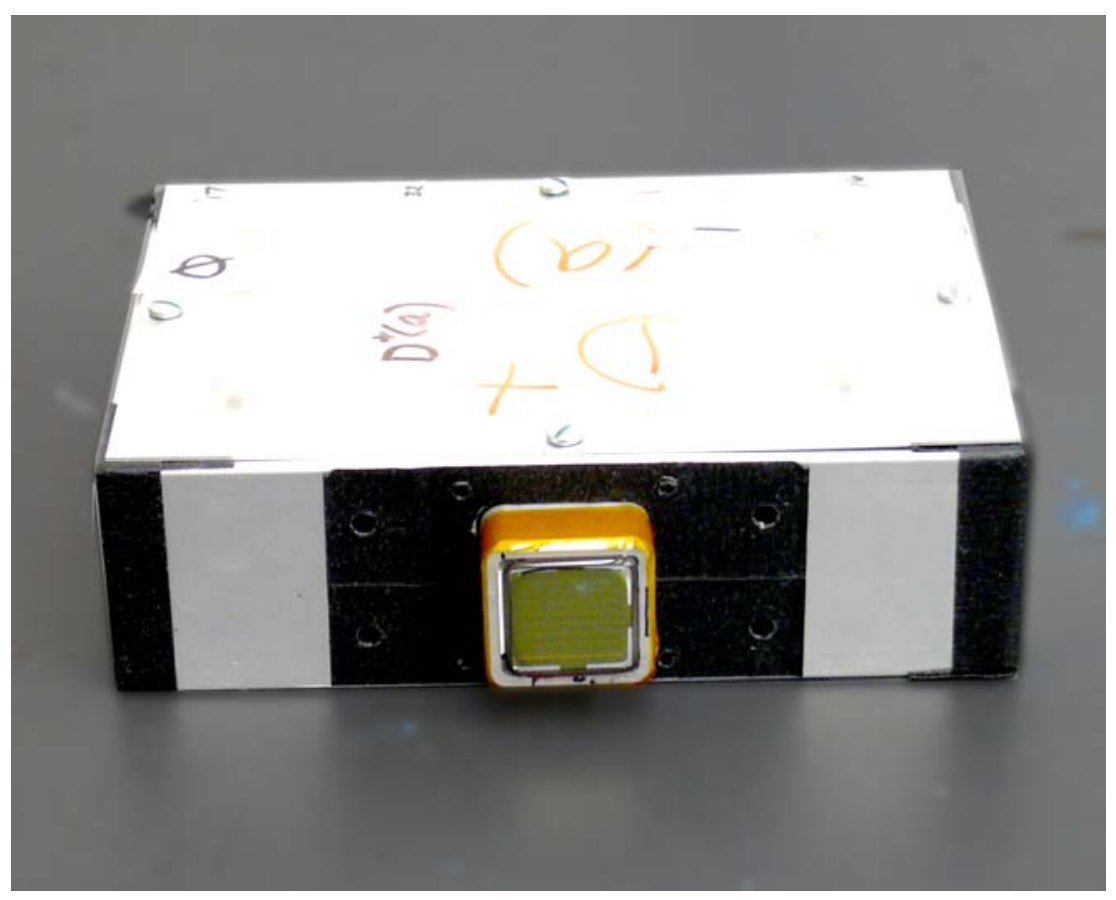

Fig.4. MAPMT assembly box. 


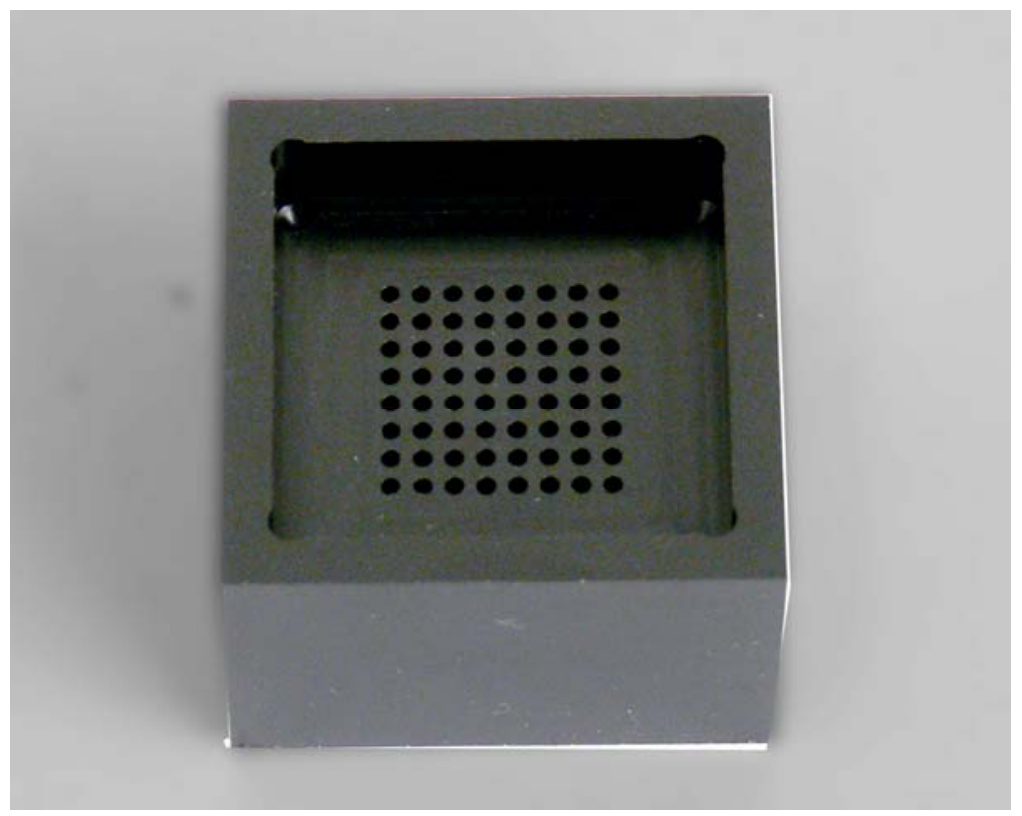

Fig.5a. A template was made from delrin [8] and had 64 holes. Each hole was about $1.3 \mathrm{~mm}$ in diameter.

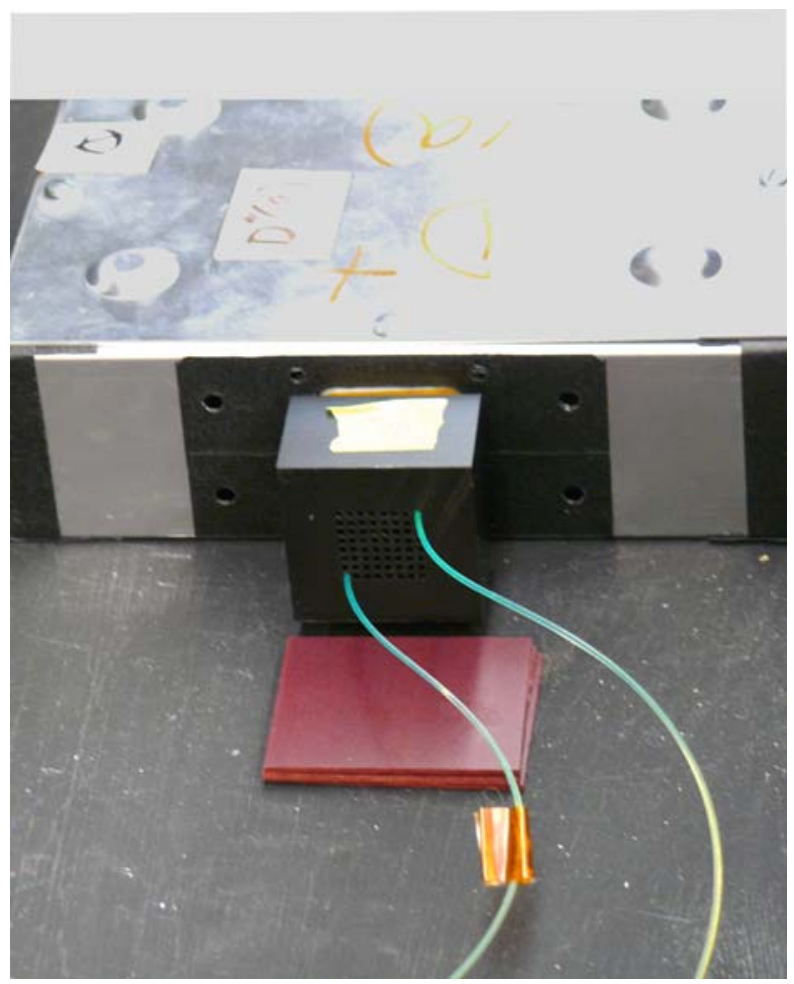

Fig.5b. A template (Fig.5a) was used to connect WLS fibers (Fig.3) to the photocathode. 


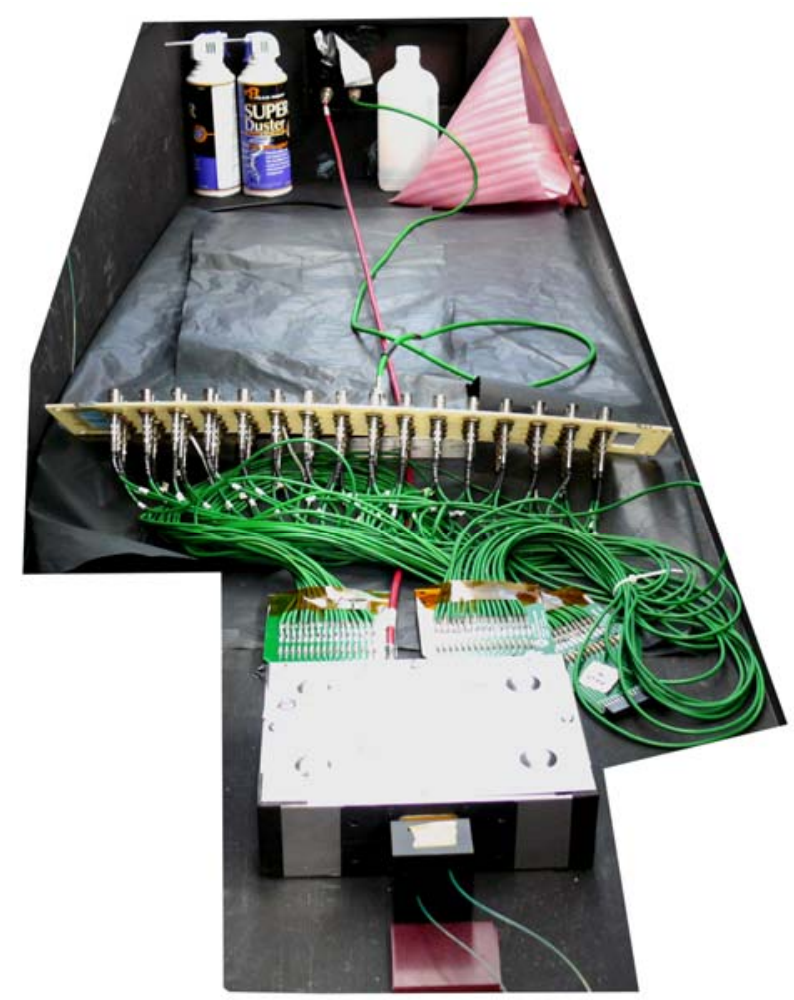

Fig.6. Each MAPMT output was measured independently using this setup.

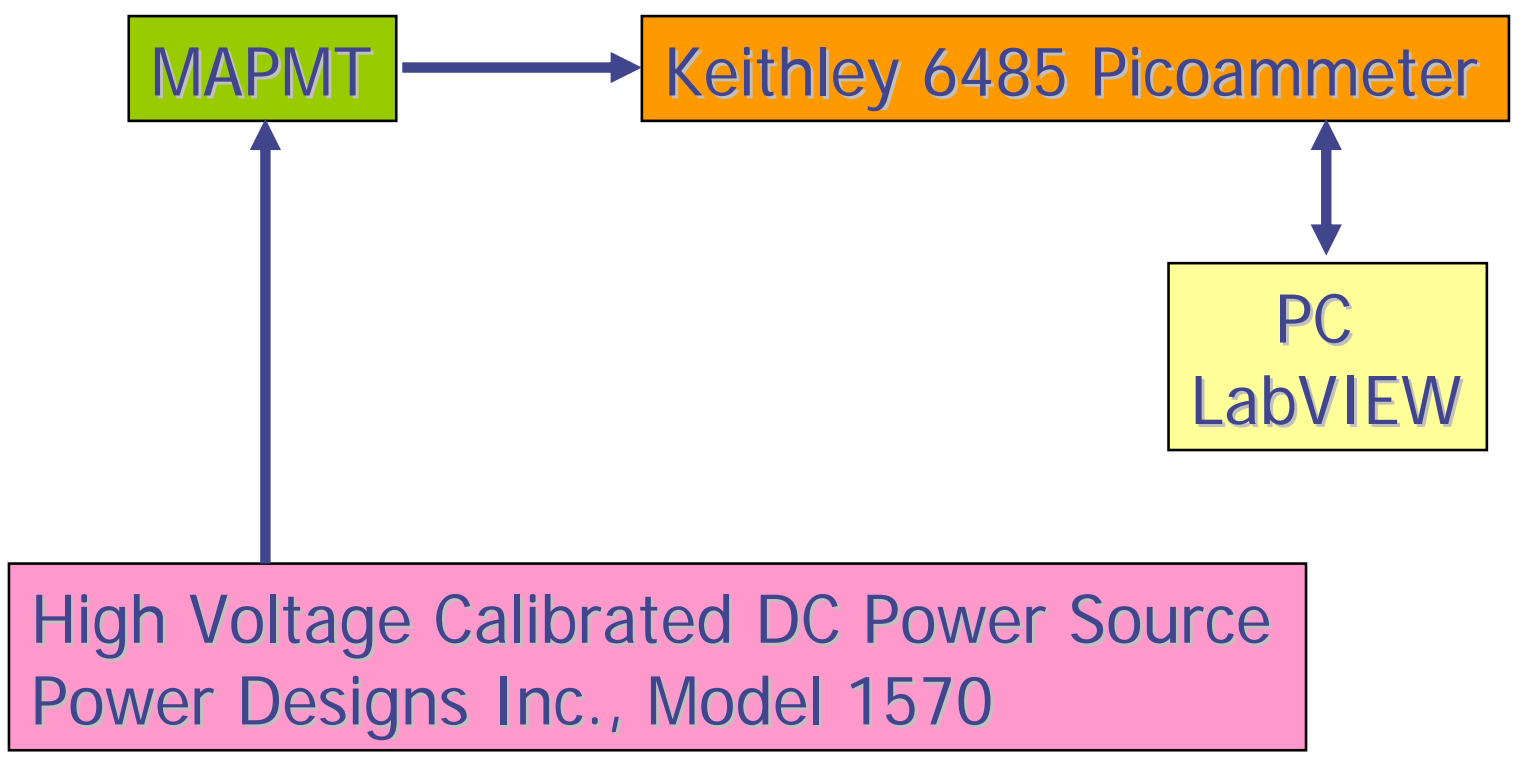

Fig.7. For the measurements a simple setup was used. 


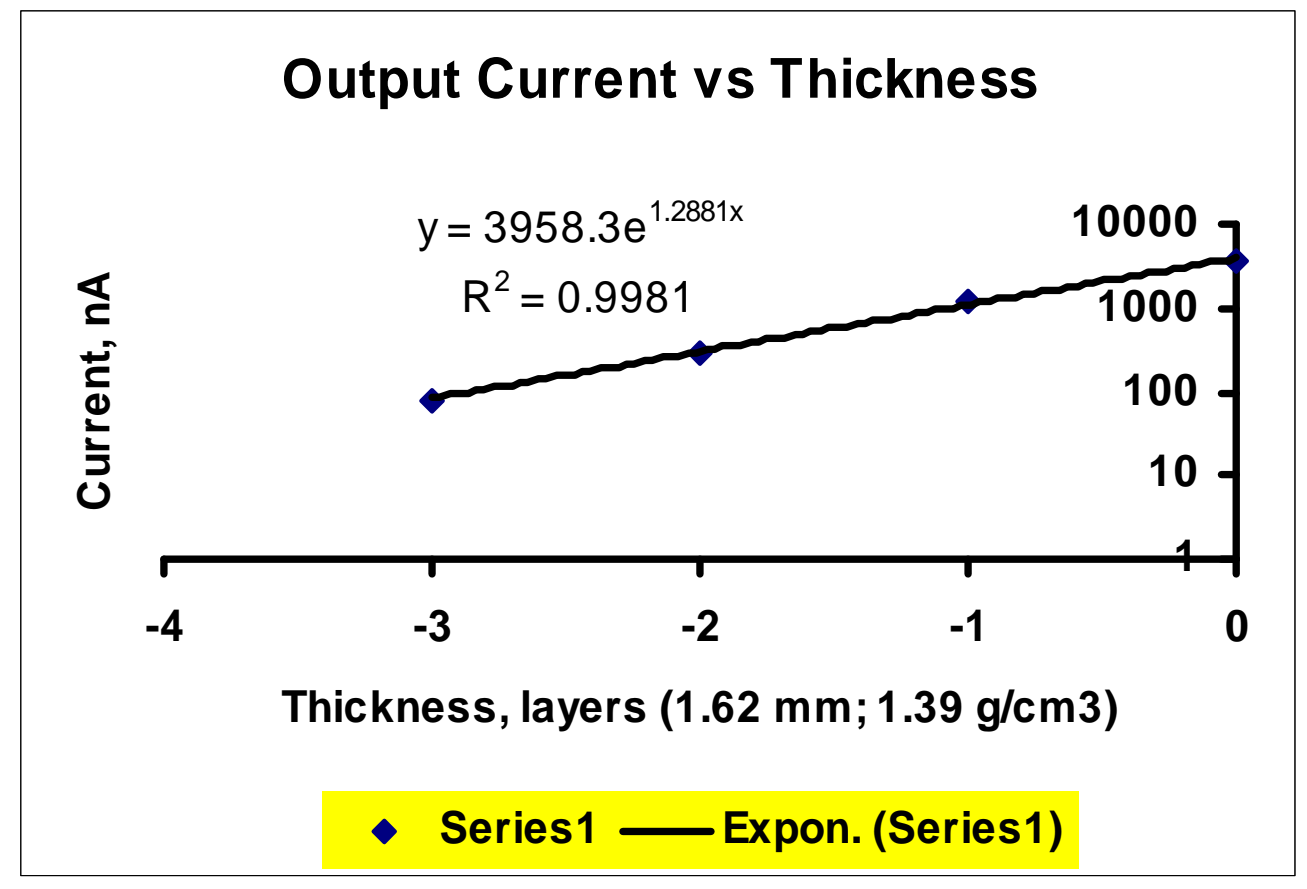

Fig.8. Verification that the brightness of the custom made light source did not saturate the anode current.

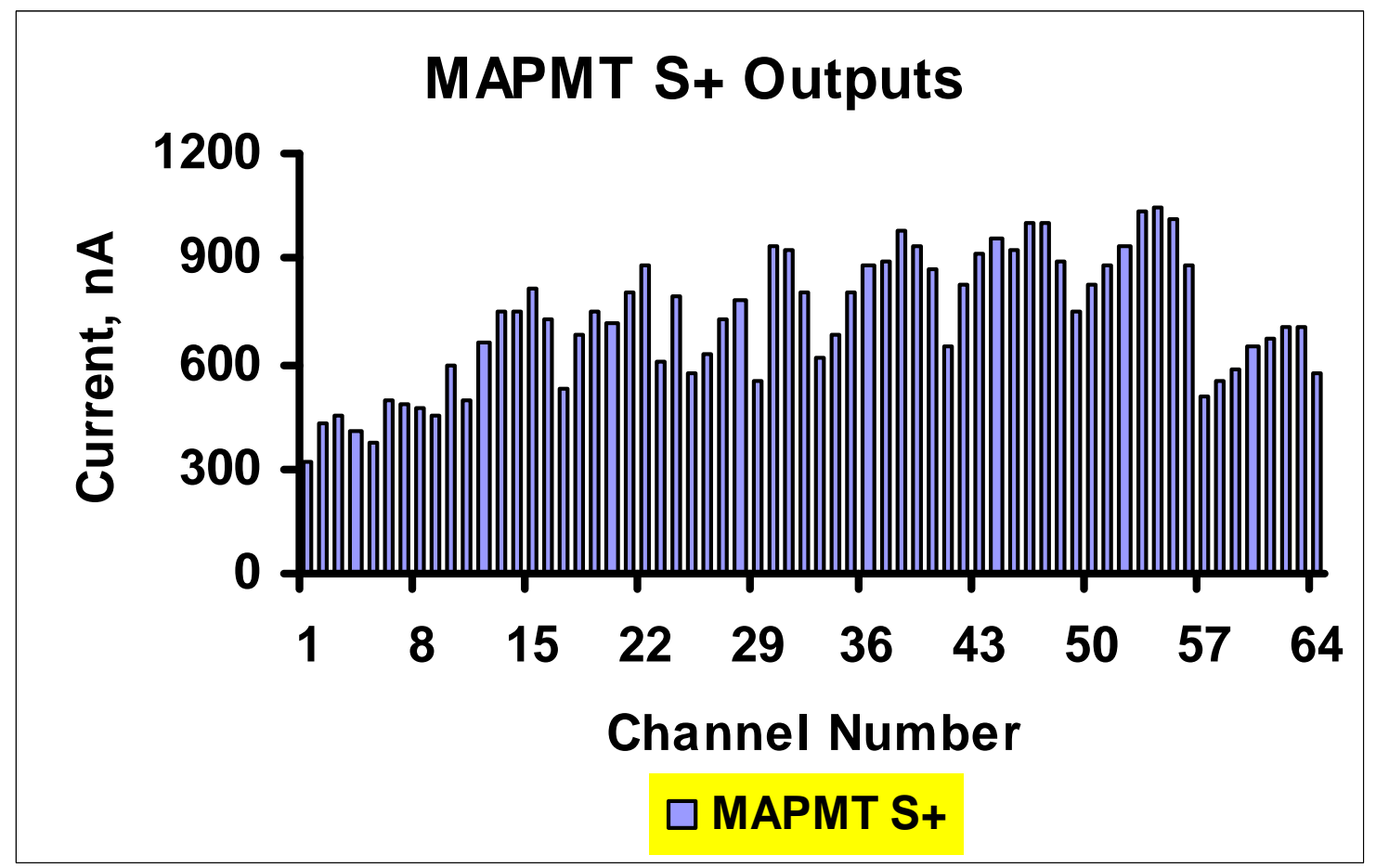

Fig.9. The anode current measured for the MAPMT S+ channels. 


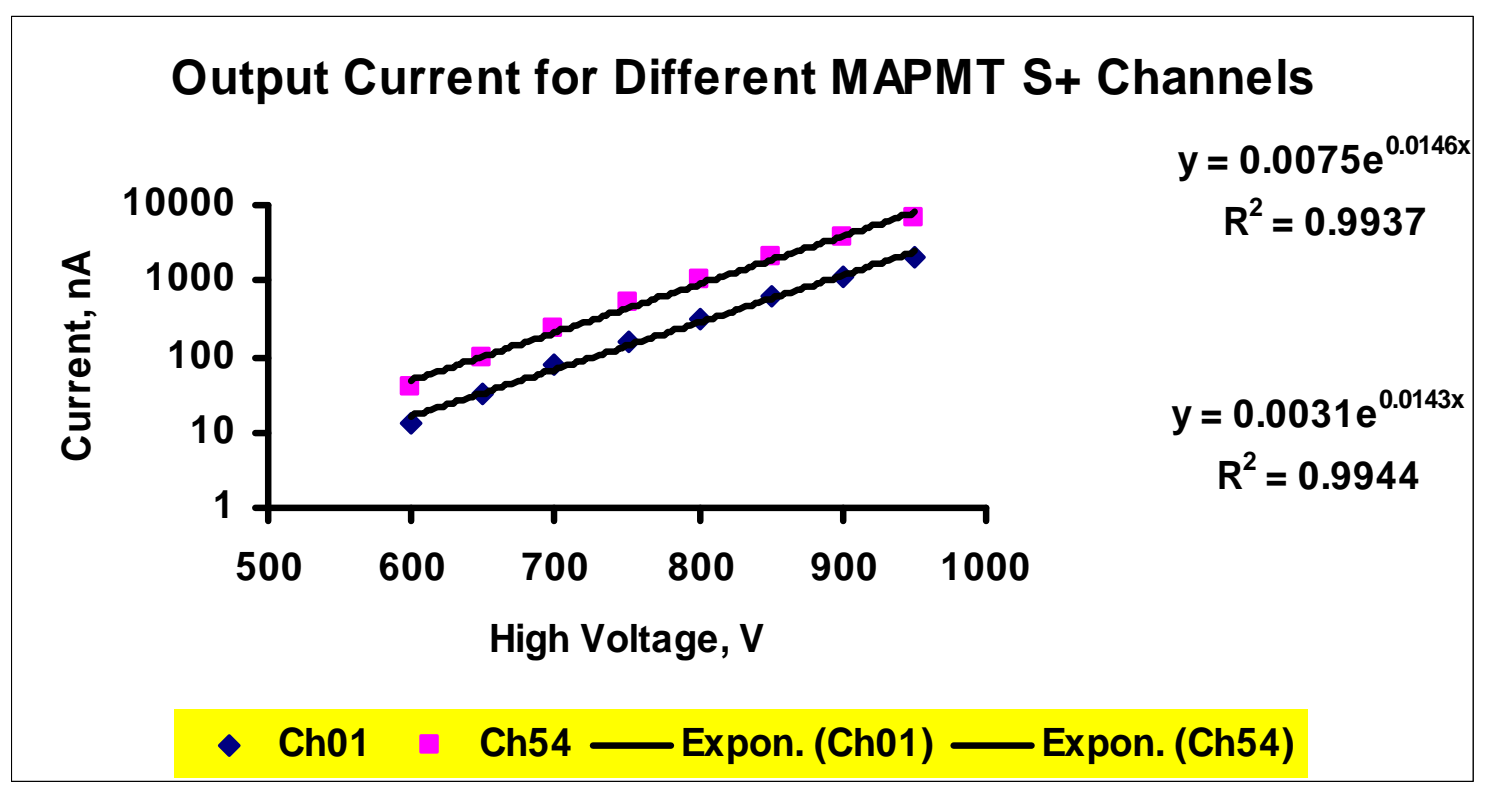

Fig.10. Anode current dependence from applied voltage for two S+ channels.

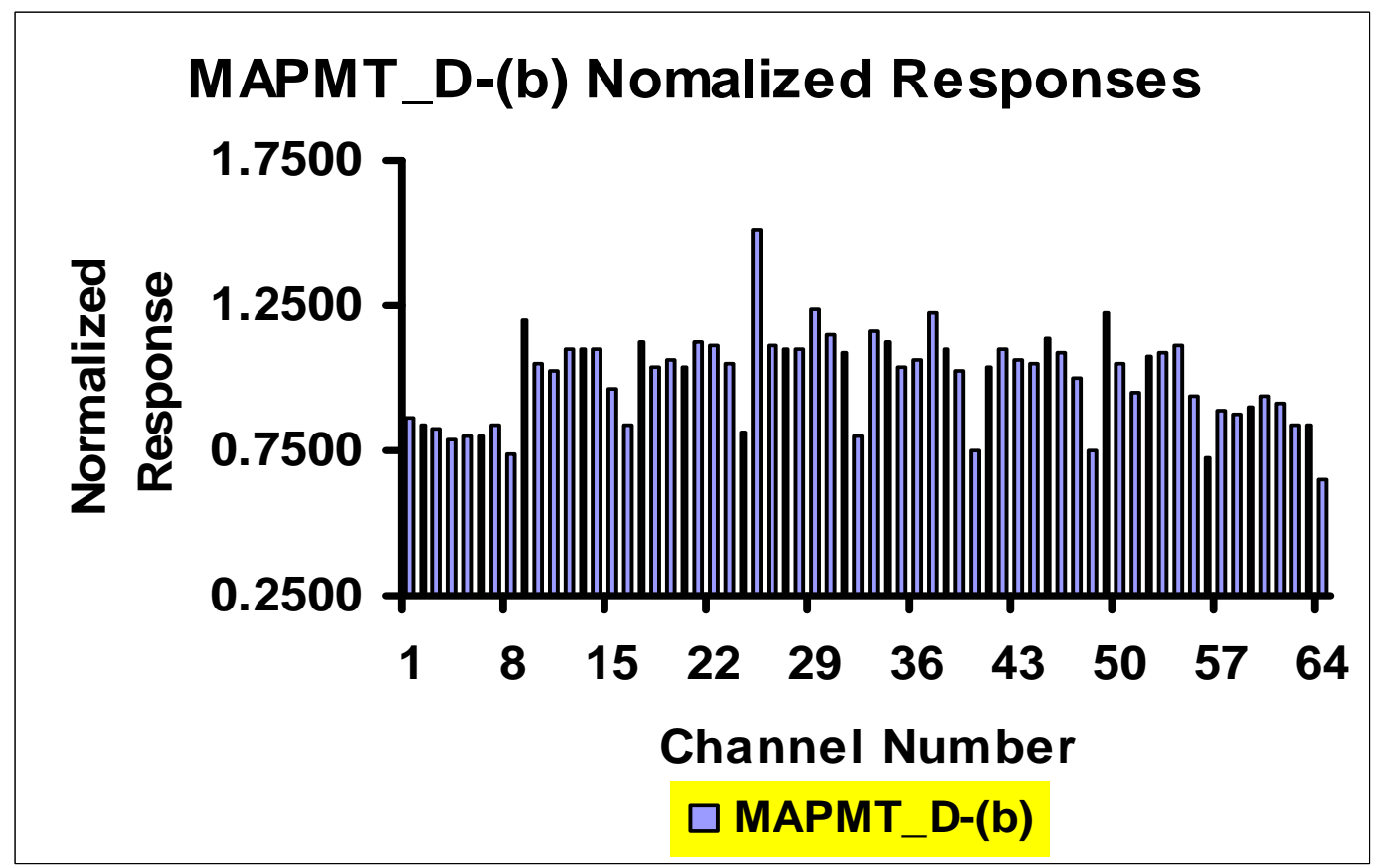

Fig.11. The response of MAPMT D-(b) channels to the same input light brightness after being normalized to the average value. 


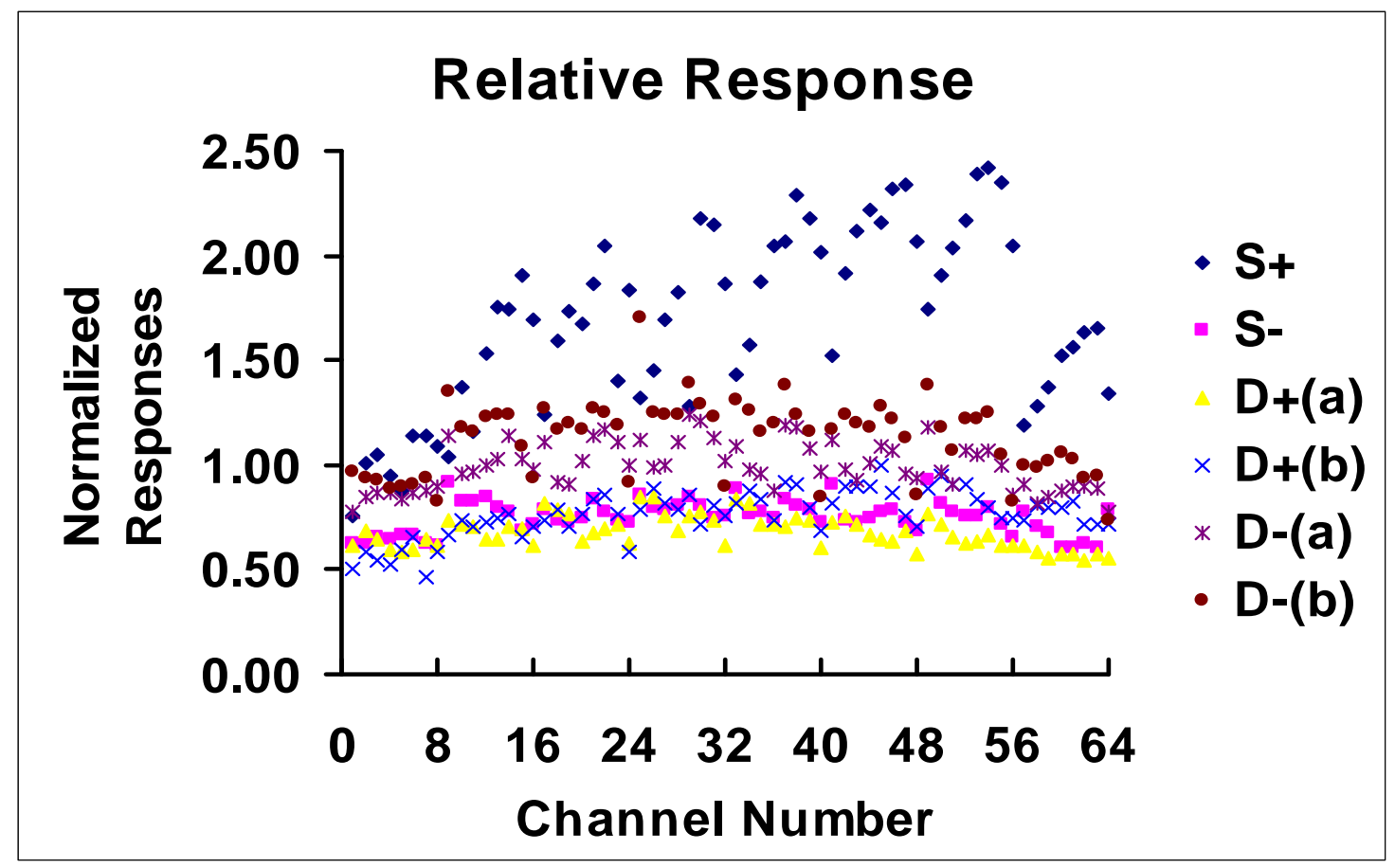

Fig.12. The distribution of all tube anode currents after being normalized to the average value.

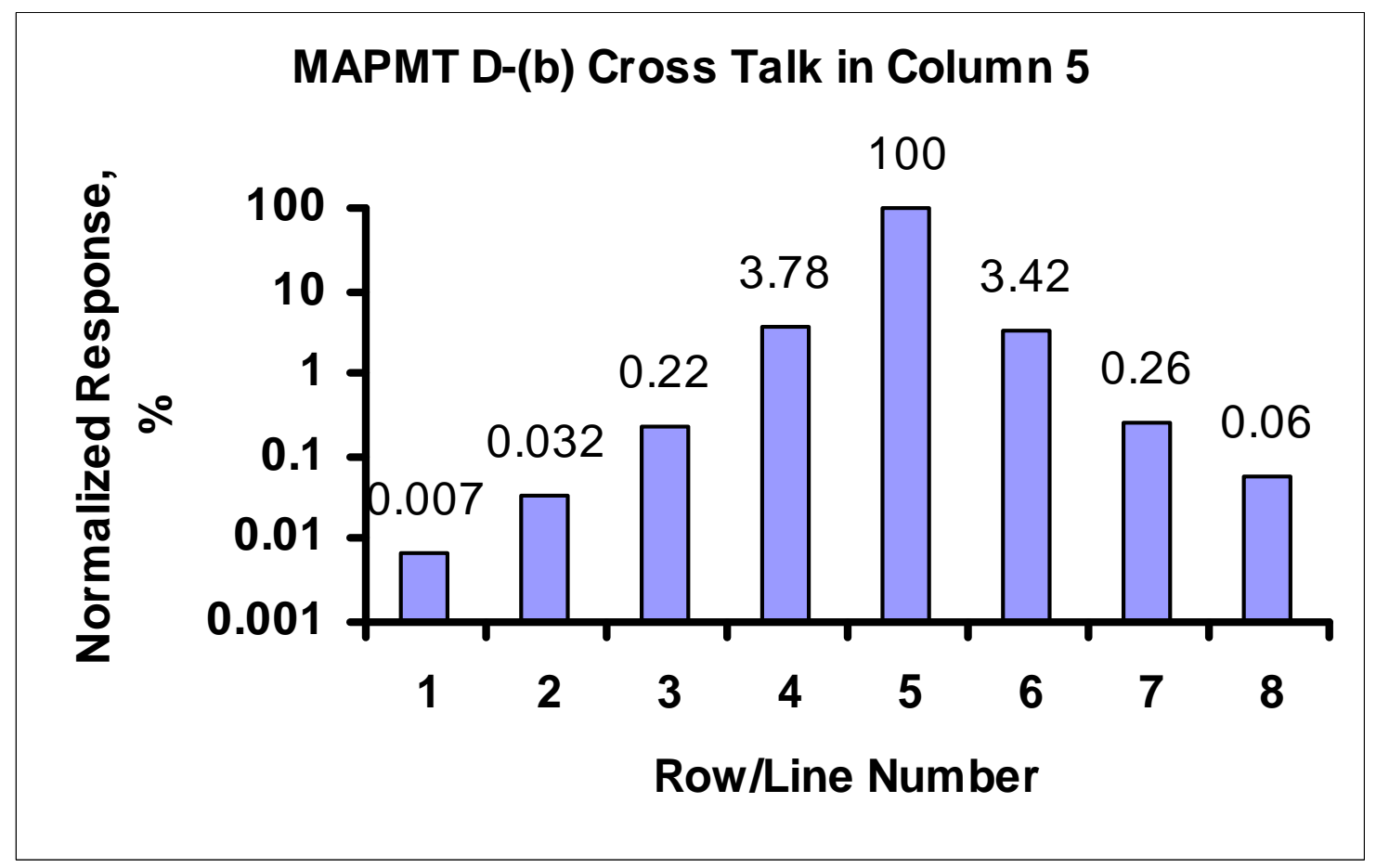

Fig.13. The distribution of MAPMT D-(b) cross talk values in column \#5 after being normalized to channel \#37. 


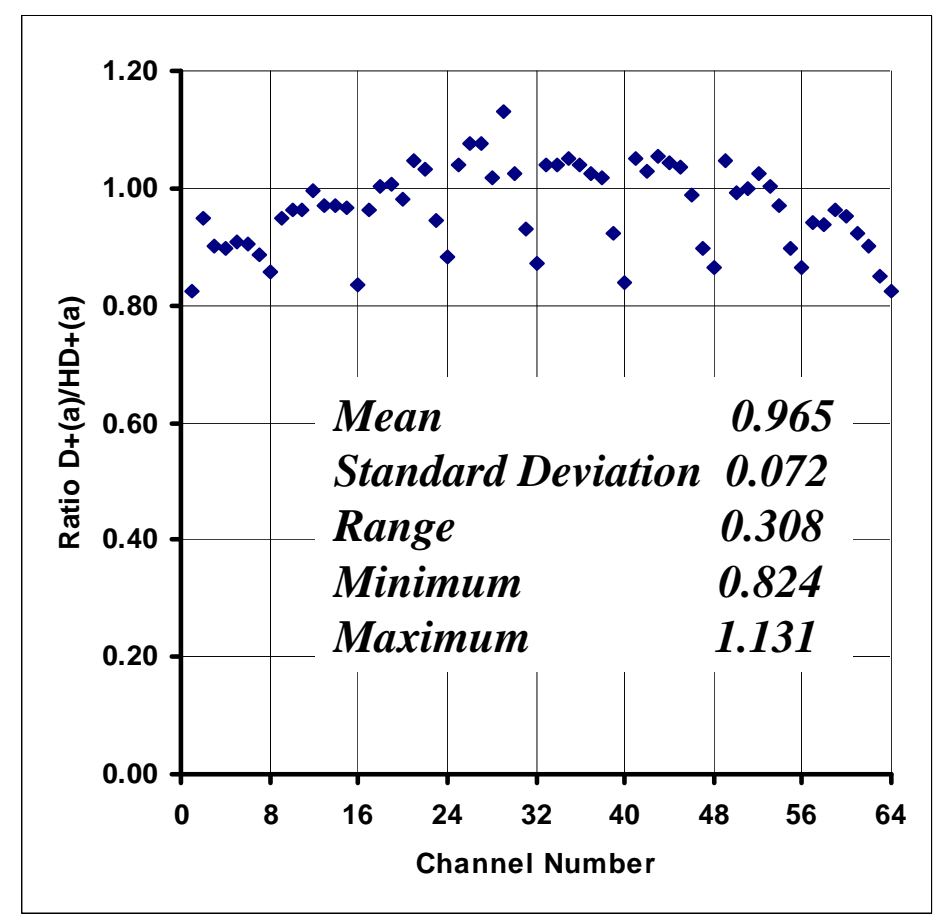

Fig.14. The ratio of $\mathrm{D}+(\mathrm{a})$ anode responses measured in this test (at about $500 \mathrm{~nm}$ ) to the Hamamatsu factory measurements HD+(a) (at about $400 \mathrm{~nm}$ ).

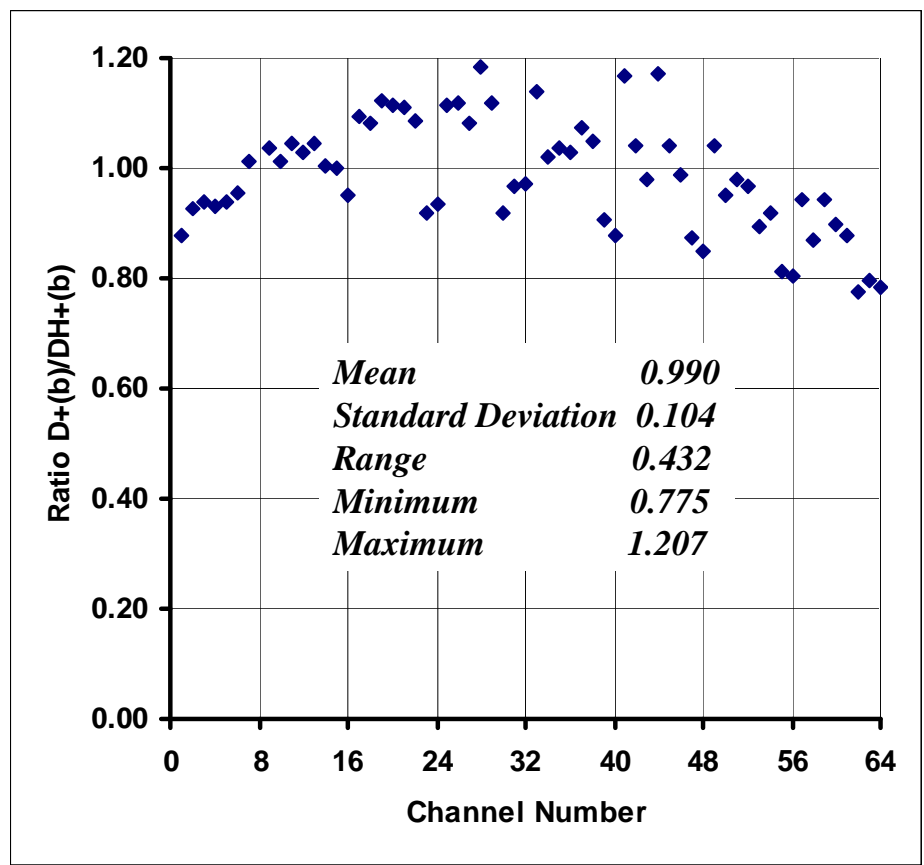

Fig.15. The ratio of $\mathrm{D}+(\mathrm{b})$ anode responses measured in this test (at about $500 \mathrm{~nm}$ ) to the Hamamatsu factory measurements $\mathrm{HD}+(\mathrm{b})$ (at about $400 \mathrm{~nm}$ ). 
Table I. The usual Hamamatsu catalog mapping that was used for inputs.

\begin{tabular}{|r|r|r|r|r|r|r|r|}
\hline 1 & 2 & 3 & 4 & 5 & 6 & 7 & 8 \\
\hline 9 & 10 & 11 & 12 & 13 & 14 & 15 & 16 \\
\hline 17 & 18 & 19 & 20 & 21 & 22 & 23 & 24 \\
\hline 25 & 26 & 27 & 28 & 29 & 30 & 31 & 32 \\
\hline 33 & 34 & 35 & 36 & 37 & 38 & 39 & 40 \\
\hline 41 & 42 & 43 & 44 & 45 & 46 & 47 & 48 \\
\hline 49 & 50 & 51 & 52 & 53 & 54 & 55 & 56 \\
\hline 57 & 58 & 59 & 60 & 61 & 62 & 63 & 64 \\
\hline
\end{tabular}

Table II. Parameters of anode current distribution (in nA).

\begin{tabular}{|l|l|l|l|l|l|}
\hline MAPMT & Mean & St.Dev. & Min & Max & Ratio \\
\hline S+ & 726.1 & 184.9 & 323.4 & 1040.5 & 3.22 \\
\hline S- & 322.2 & 34.2 & 258.8 & 400 & 1.55 \\
\hline D+(a) & 291.0 & 33.0 & 235 & 362.7 & 1.54 \\
\hline D+(b) & 328.5 & 48.0 & 198.9 & 427.5 & 2.15 \\
\hline D-(a) & 427.7 & 49.3 & 332.3 & 532.1 & 1.60 \\
\hline D-(b) & 484.6 & 76.3 & 315.4 & 731.3 & 2.32 \\
\hline
\end{tabular}


Table III. The ratios (in \%) of each anode current value as normalized to the anode current in channel \#37. The inputs mapping are as in Table 1.

\begin{tabular}{|l|l|l|l|l|l|l|l|}
\hline 0.002 & 0.003 & 0.009 & 0.008 & 0.007 & 0.006 & 0.005 & 0.005 \\
\hline 0.002 & 0.002 & 0.009 & 0.023 & 0.032 & 0.025 & 0.008 & 0.002 \\
\hline 0.009 & 0.02 & 0.06 & 0.15 & 0.22 & 0.12 & 0.04 & 0.01 \\
\hline 0.05 & 0.07 & 0.17 & 0.96 & 3.78 & 0.9 & 0.15 & 0.04 \\
\hline 0.08 & 0.1 & 0.32 & 3.53 & 100 & 4.89 & 0.2 & 0.08 \\
\hline 0.08 & 0.09 & 0.15 & 1.14 & 3.42 & 0.92 & 0.15 & 0.07 \\
\hline 0.02 & 0.1 & 0.12 & 0.15 & 0.26 & 0.19 & 0.07 & 0.04 \\
\hline 0.02 & 0.05 & 0.06 & 0.07 & 0.06 & 0.05 & 0.04 & 0.02 \\
\hline
\end{tabular}

